\title{
Os efeitos psicológicos do distanciamento social causado pelo novo Coronavírus em estudantes universitários
}

\author{
The psychological effects of social distancing caused by the new Coronavirus in university students \\ Los efectos psicológicos del distanciamiento social provocado por el nuevo Coronavirus en
} estudiantes universitarios

Recebido: 16/06/2021 | Revisado: 21/06/2021 | Aceito: 29/06/2021 | Publicado: 05/07/2021

\author{
Ana Flávia Machado de Carvalho \\ ORCID: https://orcid.org/0000-0002-6691-4804 \\ Centro Universitário Unifacid, Brasil \\ E-mail: anaflaviaparaibana@htomail.com \\ Gleysiane Gonçalves de Sousa \\ ORCID: https://orcid.org/0000-0002-0275-6886 \\ Centro Universitário Unifacid, Brasil \\ E-mail: gleysianegs@gmail.com
}

\begin{abstract}
Resumo
As medidas de distanciamento social implementadas para frear a propagação do novo coronavirus podem ter um resultado desastroso e desencadear efeitos psicológicos negativos. Os estudantes universitários parecem estar sendo particularmente impactados pelas restrições decorrentes do COVID-19. Nesse cenário, o objetivo do presente estudo foi analisar os efeitos psicológicos do distanciamento social decorrente do COVID-19 em estudantes de graduação. Trata-se de uma revisão narrativa da literatura, realizada a partir da busca de artigos científicos nas bases de dados PubMed, Scielo e Science Direct, publicados no período de 2020 e 2021, usando os descritores "Coronavirus", "Covid-19", "Social Isolation", "Mental Health" e "Students". Os resultados sugerem que estudantes universitários já apresentam tendência a desenvolverem transtornos psiquiátricos e que o surto de COVID-19 acentuou ainda mais o sofrimento mental nesta população, indicando que o distanciamento social decorrente da pandemia atual pode ter forte influência sobre a saúde psicológica e que mulheres e pessoas mais jovens têm risco aumentado para o desenvolvimento de transtornos. No entanto, não é possível concluir que, por si só, o distanciamento social seja capaz de induzir ao sofrimento psíquico, denotando a necessidade de maiores estudos que considerem este aspecto.
\end{abstract}

Palavras-chave: Coronavírus; COVID-19; Isolamento social; Saúde mental; Estudantes.

\begin{abstract}
Social distancing measures implemented to curb the spread of the new coronavirus can have a disastrous result and trigger negative psychological effects. College students seem to be particularly impacted by the restrictions arising from COVID-19. In this scenario, the aim of this study was to analyze the psychological effects of social distancing resulting from COVID-19 in undergraduate students. This is a narrative review of the literature, carried out from the search for scientific articles in the PubMed, Scielo and Science Direct databases, published in the period 2020 and 2021, using the descriptors "Coronavirus", "Covid-19", "Social Isolation", "Mental Health" and "Students". The results suggest that university students already have a tendency to develop psychiatric disorders and that the outbreak of COVID-19 further accentuated mental suffering in this population, indicating that the social distance resulting from the current pandemic can have a strong influence on psychological health and that women and younger people are at increased risk for developing disorders. However, it is not possible to conclude that, by itself, social distancing is capable of inducing psychological distress, denoting the need for further studies that consider this aspect.
\end{abstract}

Keywords: Coronavirus; COVID-19; Social isolation; Mental health; Students.

\section{Resumen}

Las medidas de distanciamiento social implementadas para frenar la propagación del nuevo coronavirus pueden tener un resultado desastroso y desencadenar efectos psicológicos negativos. Los estudiantes universitarios parecen verse particularmente afectados por las restricciones derivadas de COVID-19. En este escenario, el objetivo de este estudio fue analizar los efectos psicológicos del distanciamiento social derivado del COVID-19 en estudiantes de pregrado. Se trata de una revisión narrativa de la literatura, realizada a partir de la búsqueda de artículos científicos en las bases de datos PubMed, Scielo y Science Direct, publicados en el período 2020 y 2021, utilizando los descriptores "Coronavirus", "Covid-19", “Social Isolation", "Mental Health" y "Students". Los resultados sugieren que los estudiantes universitarios ya tienen una tendencia a desarrollar trastornos psiquiátricos y que el brote de COVID-19 acentuó aún más el sufrimiento mental en esta población, lo que indica que la distancia social resultante de la 
pandemia actual puede tener una fuerte influencia en la salud psicológica y que las mujeres y las personas más jóvenes tienen un mayor riesgo de desarrollar trastornos. Sin embargo, no es posible concluir que, por sí solo, el distanciamiento social sea capaz de inducir malestar psicológico, lo que denota la necesidad de más estudios que consideren este aspecto.

Palabras clave: Coronavirus; COVID-19; Aislamiento social; Salud mental; Estudiantes.

\section{Introdução}

Em função do surto que se instalou com o COVID-19, estamos vivendo tempos sem precedentes na história moderna (Fauci, Lane \& Redfield, 2020). O aumento na quantidade de países afetados gerou uma preocupação pública em infectar-se, dada a imprevisibilidade dessa pandemia que foi exacerbada por mitos e desinformação, além das proibições de viagens e imposição de medidas de quarentena que podem ter gerado ansiedade nas pessoas ao tentar conter a disseminação da doença (Bao, Sun, Meng, Shi \& Lu, 2020).

Esse cenário tem se caracterizado como um estressor psicológico significativo, pois além do impacto na vida das pessoas, nas organizações, nos aspectos sociais e econômicos em todo o mundo, o medo de ser contaminado e as incertezas sobre o futuro, precipitam distúrbios psicológicos (Troyer, Kohn \& Hong, 2020). A atual pandemia é considerada uma fonte potencial de traumatização direta e indireta para todos ( $\mathrm{Li}$ et al., 2020), mas parece que estudante de ensino superior foram especialmente afetados (Wathelet et al., 2020).

É possível que os efeitos psicológicos das medidas de distanciamento social tenham consequências de longo prazo e para mitigar as consequências negativas, é importante a identificação dos indivíduos em risco e a realização de intervenções não apenas durante, mas também após a remoção das medidas de distanciamento (Lopez-Carral, Grechuta \& Verschure, 2020).

Dado que a saúde mental de estudantes universitários é tida como uma preocupação de saúde pública que se tornou ainda mais crítica no cenário de pandemia (Son, Hegde, Smith, Wang \& Sasangohar, 2020), o objetivo deste artigo é analisar a produção científica disponível e identificar quais são os efeitos psicológicos do distanciamento social causado pelo novo coronavírus em estudantes universitários.

\section{Metodologia}

Este é um estudo de revisão de literatura, referente à fundamentação teórica a ser assumida para investigação do problema em questão, com base na metodologia de pesquisa bibliográfica, que se norteia pela literatura já publicada (Martins, 2018). Para fundamentar este estudo, foram utilizados artigos científicos publicados nas bases de dados PubMed, Scielo e Google Scholar, no período de 2020 a 2021, usando os termos "Coronavirus" OR "Covid-19" AND "Social Isolation" AND "Mental Health" AND "Students" como descritores de assunto. Através dessa busca realizada em 31 de maio de 2020 foram encontrados 95 artigos e, após a exclusão dos que não estavam disponíveis na íntegra ou fugiam a temática proposta, 75 artigos foram analisados, restando 35 estudos com resultados significativos para embasar as discussões que se seguem.

\section{Resultados e Discussão}

\section{Impactos causados pelo COVID-19}

Medidas para limitar a mobilidade e as interações sociais foram realizadas mundialmente com a finalidade de controlar a pandemia. Essas intervenções tem o efeito de redução do número de infecções e das taxas de mortalidade, mas apesar da eficácia, são economicamente tão dispendiosas que se tornam insustentáveis no longo prazo (Giovanetti et al., 2021).

Como consequência das paralizações prolongadas, o surto de COVID-19 resultou em perda de meios de subsistência, causando um efeito cascata na economia global. Frear a disseminação contínua do SARS-CoV-2 tornou-se uma questão de 
inquietação crescente, já que o vírus continua causando prejuízos em todo o mundo, com segunda ou terceira onda de surtos sendo enfrentada por vários países, sobretudo pelo surgimento de variantes e mutações da forma viral (Cascella et al., 2021).

De acordo com Montemurro (2020), diante de um vírus com o qual a população pode não estar familiarizada, sentimentos de angústia e ansiedade podem ocorrer mesmo em pessoas sem alto risco de adoecer e, o medo de ser infectado ou morrer, assim como a sensação de desamparo, podem levar a um aumento nas taxas de suicídio.

Outros estudos já indicavam um amplo e profundo espectro de impacto psicológico que os surtos podem causar às pessoas (Lima et al., 2020), especialmente porque durante momentos como esse, as pessoas podem perder seus empregos e ter que enfrentar instabilidade habitacional, insegurança alimentar, sobrecarga pela intensificação dos cuidados com crianças ou familiares e violência doméstica, além dos indivíduos já afetados por doenças mentais provavelmente precisarem de mais apoio do que o habitual, quando sistemas de saúde mental correm o risco de perder capacidade devido ao transbordamento de hospitais e fechamento de clínicas para promover o distanciamento social (Smith et al., 2020).

O intenso trabalho dos jornalistas e das autoridades, a nível global, para divulgar informações sobre o novo coronavírus pode levar ao sofrimento psicológico resultante da exposição repetida da mídia ao surto. Isso tem implicações não apenas no sofrimento imediato em uma população que já enfrenta consequências sociais e econômicas sem precedentes, mas também nos efeitos posteriores na saúde física e mental ao longo do tempo (Garfin, Silver \& Holman, 2020).

A extensão do impacto físico, psicológico e socioeconômico da pandemia ainda precisa ser compreendido, mas o efeito potencial do COVID-19 na saúde mental não tem sido considerado adequadamente e, é muito provável que esta pandemia se torne, globalmente, o maior desafio para os sistemas de saúde (Baker \& Clark, 2020).

\section{COVID-19 e os efeitos psicológicos das medidas de distanciamento social}

O surto de doença por coronavírus que se instalou a partir de dezembro de 2019, levou muitos países a solicitarem que as pessoas que entraram em contato com a infecção se isolassem em casa ou em uma instalação de quarentena dedicada (Brooks et al., 2020). Mas com o avanço do COVID-19 pelo mundo, uma série de medidas restritivas foram impostas para limitar a propagação do vírus em níveis micro e macro (Baker \& Clark, 2020).

Medidas extremas, incluindo isolamento de grandes cidades, fechamentos de fronteiras e o confinamento das pessoas em suas residências foram implementadas (Yang, Wang, Liu, Li \& Jiang, 2021) com o surgimento do novo coronavírus, que criou uma situação confusa, onde a superlotação dos hospitais e a escassez de alimentos passou a ser fonte de preocupação, mas esse medo parece ser mais uma consequência da quarentena em massa, que pode aumentar substancialmente resposta de ansiedade na população, mesmo após o fim das medidas que limitam o contato social (Lima et al., 2020).

De acordo com Brooks et al. (2020), apesar de medidas restritivas serem necessárias, os estudos mostram que o confinamento, a perda da rotina habitual e os contatos social e físico reduzidos, frequentemente causam tédio, frustração e uma sensação de isolamento em relação ao resto do mundo, por isso precisam ser tratadas com cuidado.

Intervenções governamentais, como imposição de distanciamento social, pretendem acima de tudo, proteger a saúde física, mas podem disseminar informações equivocadas, com consequências para o bem-estar psicológico em populações vulneráveis (Xiao et al., 2020). Entretanto, qualquer indivíduo em situação de confinamento estar suscetível a prejuízos na saúde mental, por isso é importante encontrar meios de manter vínculos afetivos e sociais significativos durante o confinamento, lançando mão da criatividade na maneira como fazer isso (Vieira, Franco, Gómez Restrepo \& Abel, 2020).

O distanciamento social tem impactos disruptivos nas necessidades humanas básicas para a conexão social e pode emergir vulnerabilidades individuais inerentes a transtornos mentais (Hagerty \& Williams, 2020). Enquanto se educa sobre a necessidade de distanciamento físico, é importante reforçar a necessidade de permanecer socialmente conectado, afim de prevenir impactos negativos do isolamento e da solidão para a saúde psicológica. Essa conexão social pode ser feita por meio 
de recursos virtuais para manter uma rede de apoio (Balasuriya, Jorgensen \& Swartz, 2021).

$\mathrm{O}$ efeito psicológico de longo prazo da pandemia e das medidas de distanciamento social ainda requer mais investigação, mas é preciso aumentar o conhecimento e promover o uso de medidas preventivas em caráter urgente para ampliar a adesão ao distanciamento social e manter o bem-estar psicológico. O papel dos profissionais de saúde é fundamental no tratamento do sofrimento mental como parte da resposta à pandemia, melhorando a conformidade com o distanciamento social e ajudando a reduzir o impacto do COVID-19 na saúde psíquica (Zhao et al., 2020).

\section{Impactos do distanciamento social em estudantes de graduação}

A saúde mental dos estudantes no ensino superior, que é uma preocupação de saúde pública crescente, foi ainda mais agravada pela atual pandemia (Wathelet et al., 2020; Son et al., 2020). A COVID-19 provocou mudanças significativas na vida dos alunos com o fechamento das universidades e transferência do ensino para a modalidade online (Wieczorek et al., 2021), além da diminuição ou eliminação das interações sociais e atividades culturais, se tornando um fator de estresse adicional para a saúde mental desta população (Kohls, Baldofski, Moeller, Klemm, \& Rummel-Kluge, 2021).

Historicamente, contextos epidêmicos desencadearam consequências significativas para a saúde mental (Jia et al., 2020) da população em geral e, sabe-se que pessoas jovens e estudantes universitários são mais suscetíveis ao desenvolvimento de transtornos psiquiátricos (Brooks et al., 2020; Markowitz et al., 2021).

Medidas de quarentena, distanciamento ou isolamento social foram associadas significativamente ao risco de transtornos mentais e estudantes universitários parecem ter sido negativamente impactados pela COVID-19 (Brooks et al., 2020; Mota et al., 2021; Yang et al., 2021) com aumento de sintomas de ansiedade, depressão e outros transtornos psiquiátricos (Wieczorek et al., 2021). Ordens de lockdown, fechamento das escolas e confinamento em casa aumentam o risco de sofrimento emocional (Kohls et al., 2021; Fruehwirth; Biswas; Perreira, 2021) nessa população.

Na perspectiva da saúde pública, as medidas de distanciamento são necessárias, mas ainda requerem a compreensão dos impactos causados em indivíduos vulneráveis (Husky, Kovess-Masfety, \& Swendsen, 2020). Os estudos em torno de estudantes universitários sugerem forte necessidade de promoção de saúde mental entre esta população durante a pandemia (Sun, Goldberg, Lin, Qiao, \& Operario, 2021) e acredita-se que intervenções para promovam cuidados com a saúde psicológica, ainda que em formato virtual, podem preencher a lacuna aberta pela pandemia do coronavírus (Kohls et al., 2021).

\section{Efeitos do distanciamento social em estudantes de graduação de diferentes países}

Nos Estados Unidos, estudos apontaram que a adesão às medidas de distanciamento social foi associada a elevação dos sintomas depressivos na população (Christensen et al., 2020). Jovens universitários norte-americanos apresentaram aumento nos níveis de estresse e ansiedade devido ao surto de COVID-19, e dentre os vários estressores identificados, a diminuição das interações estava presente em $86 \%$ dos casos. A longa duração da pandemia e recomendações para ficar em casa, trouxe impactos negativos no ensino superior, destacando a necessidade de intervenções e estratégias preventivas que abordem a saúde mental de estudantes universitários (Son et al., 2020).

Uma pesquisa feita em uma grande universidade pública da Carolina do Norte (EUA), usando dados longitudinais de 419 estudantes com idades entre 18 e 20 anos, comparou o estado de saúde mental dos alunos antes do surto de COVID-19 (outubro de 2019 à fevereiro de 2020) com dados depois do início da pandemia (junho e julho de 2020). Comparativamente, os resultados mostraram um aumento na prevalência da ansiedade moderada-grave de $18,1 \%$ para $25,3 \%$, e na prevalência da depressão moderada-grave de $21,5 \%$ para $31,7 \%$, revelando que as dificuldades associadas à modalidade de ensino remoto e o distanciamento social contribuíram para o aumento desses sintomas. (Fruehwirth et al., 2021).

Outro estudo chinês conduzido com 521 estudantes universitários residentes na província de Sichuan, durante os 
meses de abril a maio de 2020, para avaliar os níveis de suspeição de transtornos mentais comuns através do teste SRQ-29, apontou indícios de sofrimento mental em 19\% dos investigados (Yang et al., 2021). Em outra pesquisa com alunos universitários da China, a prevalência de sintomas psiquiátricos foi ainda mais alarmante, revelando estresse traumático em $67,05 \%$, sintomas depressivos em 46,55\%, sintomas de ansiedade em 34,73\% e ideação suicida em 19,56\% dos participantes (Sun et al., 2021).

Na Itália, Romeo et al. (2021) realizaram um estudo para investigar os efeitos psicológicos do COVID-19 em alunos de graduação comparados a trabalhadores em geral e verificaram que o impacto psicológico encontrado entre os estudantes pode ter sido influenciado pelo isolamento social. A presença de níveis mais elevados de ansiedade e depressão foi destacada nos alunos, entre os quais fatores significativos para predizer os índices elevados desses sintomas foram ser do sexo feminino, ter uma classificação mais baixa da própria saúde, estar mais preocupado em ser infectado e ter nível educacional mais baixo.

Uma pesquisa na França com mais de 69 mil estudantes que vivenciaram a quarentena, verificou taxas elevadas na prevalência de sintomas graves de saúde psicológica e foram identificados entre os fatores de risco com associação para alteração na condição mental, sexo feminino ou não binário, questões relacionadas à renda ou moradia, histórico de problemas psiquiátricos, baixa qualidade de informações recebidas sobre o novo vírus, sintomas de COVID-19 e isolamento social (Wathelet et al., 2020).

Na Espanha, um estudo desenvolvido para analisar os efeitos psicológicos do COVID-19 na comunidade acadêmica de uma universidade durante as primeiras semanas de confinamento no país, revelou que os estudantes foram especialmente impactados pelas medidas de lockdown e foram encontrados escores moderados a extremamente graves de ansiedade, depressão e estresse em parcela significativa dos entrevistados (Odriozola-González, Planchuelo-Gómez, Irurtia, \& de LuisGarcía, 2020).

Outra pesquisa conduzida com estudantes universitários espanhóis por Garvey et al. (2021), para averiguar como estavam se sentindo após um mês de rígido confinamento, identificou que 18,7\% deles sofriam de ansiedade severa e 70,2\% apresentavam sintomas de ansiedade em grau leve ou moderado, tendo as mulheres expressado os níveis mais elevados.

Wieczorek et al. (2021), constaram em um estudo realizado na Polônia com estudantes de graduação que, alunos do primeiro ano e mulheres tinham risco aumentado para transtornos psiquiátricos. De acordo com os autores, as universidades tem um importante papel no desenvolvimento da identidade e sentimento de pertencimento dos jovens na sociedade, portanto devem assumir o desafio de não apenas continuar com a transmissão do conhecimento de forma eficiente, mas também garantir a continuidade da construção da comunidade estudantil e das relações professor-aluno por meio das novas tecnologias.

$\mathrm{Na}$ Alemanha, um estudo transversal com 3.382 estudantes universitários para examinar o bem-estar social e emocional durante o lockdown decorrente do COVID-19, descobriu que 49\% dos alunos estavam preocupados ou muito preocupados com a pandemia e, apesar da maioria (85\%) apoiar as medidas de restrição de contato social, $37 \%$ deles apresentou sintomas depressivos clinicamente relevantes e 14,5\% revelou pensamentos suicidas, indicando que esta é uma população vulnerável, com sintomas de depressão elevados e que foi fortemente afetada pelo surto do novo coronavírus (Kohls et al., 2021).

Um estudo brasileiro com acadêmicos de medicina da região de Carajás para examinar o impacto psicológico da pandemia, encontrou desordens psiquiátricas em 43,4\% dos participantes. A ansiedade foi o traço mais prevalente $(23,1 \%)$, seguido de transtorno ansioso e depressivo simultaneamente (11,5\%) e depressão $(8,8 \%)$. A maior prevalência de problemas psicológicos foi associada ao sexo feminino. (Silva et al., 2020).

Outra pesquisa realizada com alunos de um centro universitário situado em Juiz de Fora, na cidade de Minas Gerais, para estimar a prevalência de transtornos mentais comuns (TCM) em uma amostra não probabilística de 275 estudantes, com idade acima de 17 anos e de ambos os sexos, durante o segundo mês de pandemia, verificou uma prevalência de TCM de 
58,5\% (Mota et al., 2021).

Em Teresina-PI, um estudo descritivo analítico foi aplicado com 11 estudantes de graduação, com idades de 20 a 28 anos, para compreender os efeitos do isolamento social e os impactos causados pela pandemia nas dimensões social, econômica, familiar e de saúde, através da percepção compartilhada pelos alunos em rodas de conversas online. Os relatos dos participantes indicam prejuízo na saúde mental como consequência do isolamento social, dificuldade em lidar com efeitos da pandemia, como redução de renda e alteração de rotina, mas sugerem ressignificação de laços familiares e de novas formas de ensino-aprendizagem (Nascimento et al., 2020).

\section{Conclusão}

A atual pandemia se tornou um grave problema de saúde pública, com consequências importantes para a saúde mental da população mundial e, estudantes universitários parecem ter sido especialmente afetados. Por meio desta revisão foi possível verificar que as medidas de isolamento e/ou distanciamento social podem ter afetado negativamente a saúde mental de alunos de graduação, mas, apesar de muitos estudos sugerirem, ainda não existem evidências suficientes para confirmar que, por si só, tais medidas atuem como fator de risco para o adoecimento psíquico, por isso é importante considerar o efeito de outros aspectos envolvidos nesse contexto.

Os níveis de sofrimento mental encontrados em estudantes universitários têm sido bastante significativos, denotando a urgência em desenvolver cuidados em saúde mental e estratégias de políticas públicas para esta população, principalmente no enfrentamento da atual pandemia. Além disso, fatores como sexo feminino e idade mais jovem parecem aumentar o risco de transtornos psiquiátricos, evidenciando a necessidade de focar em grupos mais vulneráveis.

Recomenda-se que sejam desenvolvidos trabalhos futuros com o objetivo de avaliar os impactos psicológicos das medidas de distanciamento social, em função da atual pandemia, em estudantes universitários também no longo prazo, bem como estudos comparativos com dados coletados antes do surto de COVID-19 e no período pós pandemia, para analisar o estado de saúde mental desse público e confrontar os resultados encontrados.

\section{Referências}

Baker, E., \& Clark, L. L. (2020). Biopsychopharmacosocial approach to assess impact of social distancing and isolation on mental health in older adults. British Journal of Community Nursing, 25(5), 231-238. https://doi.org/10.12968/bjen.2020.25.5.231.

Balasuriya, L., Jorgensen, S., \& Swartz, M. S. (2021). Prevention in the Era of COVID-19 and the Role of Behavioral Health Care Teams. Psychiatric Services, 72(4), 369-369. https://doi.org/10.1176/appi.ps.72401.

Bao, Y., Sun, Y., Meng, S., Shi, J., \& Lu, L. (2020). 2019-nCoV epidemic: address mental health care to empower society. The Lancet, 395(10224), e37-e38. https://doi.org/10.1016/S0140-6736(20)30309-3.

Brooks, S. K., Webster, R. K., Smith, L. E., Woodland, L., Wessely, S., Greenberg, N., \& Rubin, G. J. (2020). The psychological impact of quarantine and how to reduce it: rapid review of the evidence. The Lancet, Vol. 395, p. 912-920. https://doi.org/10.1016/S0140-6736(20)30460-8.

Cascella, M., Rajnik, M., Aleem, A., Scott, C., Napoli, R. Di, Mariano, V., \& Network, H. (2021). Features, Evaluation, and Treatment of Coronavirus (COVID-19). StatPearls Publishing.

Christensen, S. R., Pilling, E. B., Eyring, J. B., Dickerson, G., Sloan, C. D., \& Magnusson, B. M. (2020). Political and personal reactions to COVID-19 during initial weeks of social distancing in the United States. PLOS ONE, 15(9 September), 1-16. https://doi.org/10.1371/journal.pone.0239693.

Fauci, A. S., Lane, H. C., \& Redfield, R. R. (2020). Covid-19 — Navigating the Uncharted. New England Journal of Medicine, $382(13)$, $1268-1269$. https://doi.org/10.1056/NEJMe2002387.

Fruehwirth, J. C., Biswas, S., \& Perreira, K. M. (2021). The Covid-19 pandemic and mental health of first-year college students: Examining the effect of Covid-19 stressors using longitudinal data. PLOS ONE, 16(3), e0247999. https://doi.org/10.1371/journal.pone.0247999.

Garfin, D. R., Silver, R. C., \& Holman, E. A. (2020). The novel coronavirus (COVID-2019) outbreak: Amplification of public health consequences by media exposure. Health Psychology, 39(5), 355-357. https://doi.org/10.1037/hea0000875.

Garvey, A. M., García, I. J., Franco, S. H. O., \& Fernández, C. M. (2021). The psychological impact of strict and prolonged confinement on business students during the COVID-19 pandemic at a Spanish university. International Journal of Environmental Research and Public Health, 18 , 1-13. 
https://doi.org/10.3390/ijerph18041710.

Giovanetti, M., Benedetti, F., Campisi, G., Ciccozzi, A., Fabris, S., Ceccarelli, G., \& Ciccozzi, M. (2021). Evolution patterns of SARS-CoV-2: Snapshot on its genome variants. Biochemical and Biophysical Research Communications, 538(January), 88-91. https://doi.org/10.1016/j.bbrc.2020.10.102.

Hagerty, S. L., \& Williams, L. M. (2020). The impact of COVID-19 on mental health: The interactive roles of brain biotypes and human connection. Brain, Behavior, \& Immunity - Health, 5, 100078. https://doi.org/10.1016/j.bbih.2020.100078.

Husky, M. M., Kovess-Masfety, V., \& Swendsen, J. D. (2020). Stress and anxiety among university students in France during Covid-19 mandatory confinement. Comprehensive Psychiatry, Vol. 102. https://doi.org/10.1016/j.comppsych.2020.152191.

Jia, R., Ayling, K., Chalder, T., Massey, A., Broadbent, E., Coupland, C., \& Vedhara, K. (2020). Mental health in the UK during the COVID-19 pandemic: cross-sectional analyses from a community cohort study. BMJ open, 10(9), e040620. https://doi.org/10.1136/bmjopen-2020-040620.

Kohls, E., Baldofski, S., Moeller, R., Klemm, S.-L., \& Rummel-Kluge, C. (2021). Mental Health, Social and Emotional Well-Being, and Perceived Burdens of University Students During COVID-19 Pandemic Lockdown in Germany. Frontiers in Psychiatry, Vol. 12. https://doi.org/10.3389/fpsyt.2021.643957.

Li, Z., Ge, J., Yang, M., Feng, J., Qiao, M., Jiang, R., \& Yang, C. (2020). Vicarious traumatization in the general public, members, and non-members of medical teams aiding in COVID-19 control. Brain, Behavior, and Immunity, 88, 916-919. https://doi.org/10.1016/j.bbi.2020.03.007.

Lima, C., PMM, C., IAAS, L., JVAO, N., JS, S., de Souza RI, \& MLR, N. (2020). The emotional impact of Coronavirus 2019-nCoV (new Coronavirus disease). Psychiatry research, Vol. 287, p. 112915. Recuperado de https://pubmed.ncbi.nlm.nih.gov/32199182/.

Lopez-Carral, H., Grechuta, K., \& Verschure, P. F. M. J. (2020). Subjective ratings of emotive stimuli predict the impact of the COVID-19 quarantine on affective states. PLoS ONE, 15(8 August), 1-15. https://doi.org/10.1371/journal.pone.0237631.

Markowitz, J. C., Milrod, B., Heckman, T. G., Bergman, M., Amsalem, D., Zalman, H., \& Neria, Y. (2021). Psychotherapy at a Distance. American Journal of Psychiatry, 178(3), 240-246. https://doi.org/10.1176/appi.ajp.2020.20050557.

Montemurro, N. (2020). The emotional impact of COVID-19: From medical staff to common people. Brain, Behavior, and Immunity, 87, 23-24. https://doi.org/10.1016/j.bbi.2020.03.032.

Mota, D. C. B., Silva, Y. V., Costa, T. A. F., Aguiar, M. H. da C., Marques, M. E. de M., \& Monaquezi, R. M. (2021). Saúde mental e uso de internet por estudantes universitários : estratégias de enfrentamento no contexto da COVID-19. Ciencia \& saude coletiva, (1678-4561).

Nascimento, E. F. do, Monte, L. M. I. do, Nascimento, M. A. C. do, Mateus, A. da S., Sousa Júnior, P. de T. X., \& Siqueira, F. I. do M. R. (2020). Juventude universitária e o isolamento social na pandemia COVID-19: Emprego, Sociabilidade e Família. Research, Society and Development, 9, e17191210995. https://doi.org/10.33448/rsd-v9i12.10995.

Odriozola-González, P., Planchuelo-Gómez, Á., Irurtia, M. J., \& de Luis-García, R. (2020). Psychological effects of the COVID-19 outbreak and lockdown among students and workers of a Spanish university. Psychiatry Research, 290. https://doi.org/10.1016/j.psychres.2020.113108.

Romeo, A., Benfante, A., Castelli, L., \& Di Tella, M. (2021). Psychological Distress among Italian University Students Compared to General Workers during the COVID-19 Pandemic. International Journal of Environmental Research and Public Health, 18(5), 2503. https://doi.org/10.3390/ijerph18052503.

Silva, A. C. da, Martins, D. da S., Santiago, A. T., Santos, O. S., Paes, C. J. O., Silva, A. C. da, \& Araújo, P. X. de. (2020). O impacto psicológico da pandemia de COVID-19 nos acadêmicos de medicina da região de Carajás / The psychological impact of the COVID-19 pandemic on medical students in the region of Carajás. Brazilian Journal of Health Review, 3(6), 19731-19747. https://doi.org/10.34119/bjhrv3n6-349.

Smith, A. C., Thomas, E., Snoswell, C. L., Haydon, H., Mehrotra, A., Clemensen, J., \& Caffery, L. J. (2020). Telehealth for global emergencies: Implications for coronavirus disease 2019 (COVID-19). Journal of Telemedicine and Telecare, 26(5), 309-313. https://doi.org/10.1177/1357633X20916567.

Son, C., Hegde, S., Smith, A., Wang, X., \& Sasangohar, F. (2020). Effects of COVID-19 on College Students' Mental Health in the United States: Interview Survey Study. Journal of medical Internet research, 22(9), e21279. https://doi.org/10.2196/21279.

Sun, S., Goldberg, S. B., Lin, D., Qiao, S., \& Operario, D. (2021). Psychiatric symptoms, risk, and protective factors among university students in quarantine during the COVID-19 pandemic in China. Globalization and Health, Vol. 17. https://doi.org/10.1186/s12992-021-00663-x.

Troyer, E. A., Kohn, J. N., \& Hong, S. (2020). Are we facing a crashing wave of neuropsychiatric sequelae of COVID-19? Neuropsychiatric symptoms and potential immunologic mechanisms. Brain, Behavior, and Immunity, 87, 34-39. https://doi.org/10.1016/j.bbi.2020.04.027.

Vieira, C. M., Franco, O. H., Gómez Restrepo, C., \& Abel, T. (2020). COVID-19: The forgotten priorities of the pandemic. Maturitas, 136, 38-41. https://doi.org/10.1016/j.maturitas.2020.04.004.

Wathelet, M., Duhem, S., Vaiva, G., Baubet, T., Habran, E., Veerapa, E., \&D’Hondt, F. (2020). Factors Associated With Mental Health Disorders Among University Students in France Confined During the COVID-19 Pandemic. JAMA Network Open, 3(10), e2025591. https://doi.org/10.1001/jamanetworkopen.2020.25591.

Wieczorek, T., Kołodziejczyk, A., Ciułkowicz, M., Maciaszek, J., Misiak, B., Rymaszewska, J., \& Szcześniak, D. (2021). Class of 2020 in Poland: Students' Mental Health during the COVID-19 Outbreak in an Academic Setting. International Journal of Environmental Research and Public Health, $18(6), 2884$. https://doi.org/10.3390/ijerph18062884.

Xiao, H., Shu, W., Li, M., Li, Z., Tao, F., Wu, X., \& Hu, Y. (2020). Social distancing among medical students during the 2019 coronavirus disease pandemic in china: Disease awareness, anxiety disorder, depression, and behavioral activities. International Journal of Environmental Research and Public Health, 17(14), 1-13. https://doi.org/10.3390/ijerph17145047.

Yang, K. H., Wang, L., Liu, H., Li, L. X., \& Jiang, X. L. (2021). Impact of coronavirus disease 2019 on the mental health of university students in Sichuan 
Research, Society and Development, v. 10, n. 8, e9710817245, 2021

(CC BY 4.0) | ISSN 2525-3409 | DOI: http://dx.doi.org/10.33448/rsd-v10i8.17245

Province, China: An online cross-sectional study. International Journal of Mental Health Nursing. https://doi.org/10.1111/inm.12828.

Zhao, S. Z., Wong, J. Y. H., Wu, Y., Choi, E. P. H., Wang, M. P., \& Lam, T. H. (2020). Social distancing compliance under covid-19 pandemic and mental health impacts: A population-based study. International Journal of Environmental Research and Public Health, 17(18), 1-11. https://doi.org/10.3390/ijerph17186692 